\title{
Calidad de los resúmenes de tesis de doctorados y maestrías de la Universidad Nacional de Asunción, 2000-2017
}

\author{
*Carmiña Soto ${ }^{1}$, María José Aparicio² \\ ${ }^{1}$ Universidad Nacional de Asunción. Dirección General de Postgrado y Relaciones Internacionales. Paraguay \\ ${ }^{2}$ Universidad Nacional de Asunción. Facultad de Ciencias Agrarias. Carrera de Ecología Humana. Paraguay
}

Cómo referenciar este artículo/

How to reference this article:
Soto C, Aparicio MJ. Calidad de los resúmenes de tesis de doctorados y maestrías de la Universidad Nacional de Asunción, 2000-2017. Mem. Inst. Investig. Cienc. Salud. 2019; 17(3): 63-71

\section{RE S U M E N}

Los resúmenes de las tesis permiten que los lectores conozcan de manera rápida el contenido de las mismas, y representan la visibilidad institucional, sin embargo, la calidad de los mismos no es analizada de manera frecuente. De allí, que el objetivo del trabajo fue relevar la calidad de los resúmenes de las tesis de maestrías y doctorados de la Universidad Nacional de Asunción. El diseño fue documental, descriptivo con componente analítico y trasversal donde la unidad de análisis quedó constituida por 164 tesis. Los hallazgos dan cuenta que las dimensiones calificadas como Muy bueno fueron Calidad del Título y Explicitación de Objetivos, para la dimensión Consistencia entre Objetivo, Resultado y Título, la calidad fue aceptable. Los resultados muestran la necesidad de mejorar la calidad de los resúmenes.

Palabras clave: calidad, resumen, tesis, doctorado, maestría.

\section{Quality of the abstracts of doctoral and master theses of the Universidad Nacional de Asunción, 2000-2017}

\section{A B S T R A C T}

The thesis abstracts allow readers to quickly know the content of the theses, and represent the institutional visibility, however, the quality of them is not analyzed frequently. Based on this, the objective was to evaluate the quality of the abstracts of the master and doctoral theses of the Universidad Nacional de Asunción. The design was documentary, descriptive cross-sectional with analytical components. The analysis unit was constituted by 164 theses. The findings show that the dimensions classified as very good were Quality of the Title and Objectives Explicitation, while for the dimension Consistency between Objective, Result and Title, the quality was acceptable. The results show the need to improve the quality of the abstracts.

Keywords: quality, abstract, thesis, doctorate, master.

\section{INTRODUCCIÓN}

Los estudios de postgrado se inician en la Universidad Nacional de Asunción (UNA) en la década de los noventa(1), llegando las maestrías y doctorados a sumar 34 programas a diciembre del año $2017^{(2)}$. Por otro lado, uno de los requisitos para la obtención del título de Magister en Ciencias (maestrías académicas o de investigación) o Doctor en Ciencias (doctorado con orientación académica) es la elaboración, defensa y aprobación de la tesis ${ }^{(3)}$. En este sentido y rescatando lo dicho por Umberto $\mathrm{Eco}^{(4)}$, la tesis (en especial la doctoral) es entendida como una investigación original con el objetivo de aportar de manera

Fecha de recepción: abril 2019. Fecha de aceptación: julio 2019

*Autor correspondiente: Carmiña Soto. Universidad Nacional de Asunción. Dirección General de Postgrado y Relaciones Internacionales. Paraguay

Email: carmina.soto@gmail.com 
significativa al acervo del conocimiento, e incluso "supone la primera ópera prima de cualquier investigador, su primer contacto con la investigación de primer nivel".

En el formato o estructura de la tesis, resulta prácticamente generalizada la exigencia de incluir el Resumen o Abstract que constituye "una representación abreviada y precisa del contenido de un documento, sin interpretación ni crítica y sin mención expresa del autor del resumen"(6), el cual debe ser autosuficiente de modo que el lector no precise remitirse al documento completo de tesis para obtener información básica de ésta. Constituye una carta de presentación del investigador pues "permite que los lectores reconozcan con rapidez el contenido para determinar su relevancia" y con ello despertar el interés en la lectura completa de una tesis o artículo $^{(7)}$. La importancia de una redacción con calidad del resumen de tesis radica en que representa la visibilidad institucional ante el mundo, mediante los repositorios digitales, que ponen a disposición - cuando menos- esta sección de la tesis, quedando la institución expuesta a la crítica pública.

Por ello la evaluación de la calidad de redacción de los resúmenes resulta vital y, sin embargo, no se realiza con frecuencia ${ }^{(7-9)}$. Toda evaluación de calidad resulta compleja, dada la dificultad para definir el constructo calidad. Sin embargo, necesariamente cuando se aborda este tema, debe realizarse una lectura crítica que es "una evaluación objetiva y crítica de las fortalezas y las debilidades de una investigación"(10,11), lo cual permite juzgar su mérito, significado, alcance y limitaciones. Asimismo, analizar críticamente, en este caso los resúmenes de tesis, posibilita hacer reflexiones acerca de la construcción de los conocimientos teóricos-prácticos aportados por los programas de maestrías y doctorados ${ }^{(12)}$.

Por otro lado, la evaluación se puede realizar desde un enfoque más práctico o aplicado donde, en el caso de las tesis doctorales, por ejemplo, la calidad y repercusión de una tesis se podría relevar de manera indiciaria a partir de la cantidad de publicaciones e investigaciones que genere ${ }^{(5)}$.

Como se mencionó, las evaluaciones a las tesis, a los tutores y a los programas no son frecuentes y por ello no son evidenciadas las deficiencias que pueden presentar por lo cual no son corregidas ${ }^{(8,9,11)}$. Sin embargo, el interés por la evaluación de las investigaciones se está expandiendo, propiciado tanto desde las mismas universidades, como por la comunidad científica y agentes externos como los cooperantes, donantes, principalmente desde las revistas científicas.

La calidad en la redacción de los resultados adoptando estilos y estructuras definidas, que paulatinamente va reemplazando a aquella desestructurada, intuitiva y auto informativa, se inicia principalmente como exigencias para las publicaciones, que a su vez comenzaron a otorgar prestigio y ranking profesional e institucional ${ }^{(11)}$.

En este orden, el resumen de tesis, como una forma de redacción de resultados de las investigaciones, debe contener información esencial que haga que el lector decida o no leer la totalidad del trabajo. Batista et al. ${ }^{(12)}$ coinciden con lo estipulado en el Manual de la Asociación Americana de Psicología ${ }^{(13)}$ (APA-por sus siglas en inglés) que el resumen debe reflejar de manera sucinta los pasos del proceso científico visualizándose el objetivo y el contenido del estudio de manera adecuada y eficiente con un número limitado de palabras e incluir cualidades, hipótesis, herramientas de recopilación de datos y hallazgos significativos de sujetos o participantes.

En esta línea el Manual de Tesis, elaborado por la Dirección General de Postgrado y Relaciones Internacionales del Rectorado de la UNA en el año 2013, adopta el estilo de resumen informativo pues debe reflejar "problema estudiado, materiales y métodos, resultados más importantes y principales conclusiones" y no debe incluir citas de literaturas, tablas, figuras ni abreviaturas convencionales"(14), no obstante, al interior de la Universidad Nacional de Asunción (UNA) no existe uniformidad en el tipo de resumen a utilizar, dado que cada unidad académica posee su propio manual. Desde una perspectiva técnica y global a nivel de Universidad, este problema tiene repercusión, pues no existen criterios claros y explícitos a la hora de formular los juicios requeridos.

Otros enfoques como la Norma NBR 6028/1980 de la Asociación Brasileira de Normas Técnicas ${ }^{(15)}$ señalan dos tipos de resúmenes: informativos e indicativos (o también llamados descriptivos). El primer tipo permite conocer el objeto, alcance o metodología, resultados, conclusiones y también ocasionalmente recomendaciones del trabajo; mientras que los resúmenes indicativos incluyen el objeto, alcance o metodología, pero no los resultados, conclusiones o recomendaciones. Por otro lado, se coincide con De Miguel ${ }^{(16)}$ cuando dice que usualmente los reglamentos de tesis de las Universidades suelen poner mayor énfasis 
en cuestiones de forma y cuestiones administrativas, antes que en las cuestiones técnicas y en especial en el contenido adecuado del resumen.

Con respecto a los antecedentes sobre la calidad de los resúmenes de tesis, se pueden mencionar estudios ${ }^{(11,12,17)}$ donde se encontraron deficiencias importantes en las características de calidad, tales como ausencia de objetivos y deficiencias en el diseño y en las técnicas de recopilación de datos, conclusiones no relacionadas con objetivos ni resultados planteados, títulos excesivamente largos, entre otros, llegándose en algunos casos a un $78,5 \%$ de títulos y resúmenes inaceptables ${ }^{(9)}$. Incluso Paravic y Burgos ${ }^{(11)}$ en una investigación sobre la Evaluación de calidad de resúmenes de tesis de un programa de magíster en enfermería, encontraron que ninguno de los resúmenes evaluados logró el máximo puntaje de calidad.

Por lo expuesto, y considerando a las universidades como nichos donde se deben generar investigaciones con rigurosa calidad, se llevó adelante este trabajo cuyo objetivo fue evaluar la calidad de los resúmenes de tesis del periodo 2000-2017, desde un análisis crítico, para lo cual se caracterizaron los resúmenes según programas, áreas del conocimiento y tipos de resumen, para posteriormente mediante una lista de cotejo evaluar la calidad en las dimensiones de título y cuerpo del resumen y finalmente analizar si hay asociación entre algunas variables. Se espera con ello, generar aportes para procesos reflexivos y participativos tendientes a mejorar la calidad de las investigaciones.

\section{MATERIALES Y MÉTODO}

La investigación corresponde a un estudio documental, descriptivo con componente analítico. La recolección de datos fue retrospectiva tomando como periodo 2000-2017 y la técnica de recolección de datos consistió en una lista de cotejo. La dimensión principal analizada fue la calidad de la tesis evaluada en dos segmentos: calidad del título y calidad del cuerpo de la tesis. Las pautas, o dimensiones utilizadas en el análisis crítico de los resúmenes de las tesis, fueron adaptadas principalmente de Bobenrieth ${ }^{(10)}$, así también se utilizó el Manual de Postgrado de la Dirección General de Postgrado de la UNA ${ }^{(14)}$ y las Normas de la Asociación Americana de Psicología ${ }^{(13)}$.Las dimensiones pueden verse en la Tabla 1.

Tabla 1: Dimensiones y categorías de evaluación de la calidad de los resúmenes de tesis

\begin{tabular}{|c|c|c|c|}
\hline Segmentos & Dimensiones & Categorías & Descripción de las categorías \\
\hline \multirow[t]{4}{*}{ Caracterización } & $\begin{array}{l}\text { Tipo de programa } \\
\text { realizado }\end{array}$ & $\begin{array}{l}\text { Maestría } \\
\text { Doctorado }\end{array}$ & $\begin{array}{l}700 \text { horas como mínimo } \\
1200 \text { horas como mínimo. }\end{array}$ \\
\hline & $\begin{array}{l}\text { Disciplinas según de } \\
\text { la Organización para } \\
\text { la Cooperación y el } \\
\text { Desarrollo Económico } \\
\text { (OCDE) }\end{array}$ & $\begin{array}{l}\text { Ciencias Naturales } \\
\text { Ingeniería y Tecnología } \\
\text { Ciencias Médicas y de la Salud } \\
\text { Ciencias Agrícolas } \\
\text { Ciencias Sociales } \\
\text { Humanidades }\end{array}$ & \\
\hline & Tipo de Resumen & Indicativo & $\begin{array}{l}\text { Contiene objetivo, alcance o } \\
\text { metodología, resultados, conclusiones y } \\
\text { también ocasionalmente } \\
\text { recomendaciones. } \\
\text { Contiene objetivo, alcance o } \\
\text { metodología pero no resultados y } \\
\text { conclusiones. }\end{array}$ \\
\hline & $\begin{array}{l}\text { Paradigma positivista } \\
\text { Paradigma } \\
\text { constructivista }\end{array}$ & $\begin{array}{l}\text { Cuantitativo } \\
\text { Cualitativo } \\
\text { Mixto }\end{array}$ & $\begin{array}{l}\text { Descriptivo, correlacional, experimental } \\
\text { Documental, Etnográfico, } \\
\text { Fenomenológico y Teoría } \\
\text { Fundamentada. } \\
\text { Cuali-cuantitativo }\end{array}$ \\
\hline $\begin{array}{l}\text { Calidad del } \\
\text { título }\end{array}$ & $\begin{array}{l}\text { Es indicativo del } \\
\text { estudio } \\
\text { Indica la especificidad } \\
\text { Es conciso } \\
\text { Usa tono afirmativo } \\
\text { Es gramaticalmente } \\
\text { correcto }\end{array}$ & $\begin{array}{l}\text { Si/No/Dudoso } \\
\mathrm{Si} / \text { No/Dudoso } \\
\mathrm{Si} / \mathrm{No} \\
\mathrm{Si} / \mathrm{No} \\
\mathrm{Si} / \mathrm{No}\end{array}$ & $\begin{array}{l}\text { Correspondencia del título con el } \\
\text { objetivo. } \\
\text { Responde a la pregunta ¿buscando qué? } \\
\text { Contiene entre } 12 \text { y } 17 \text { palabras. } \\
\text { Redactado en forma afirmativa. } \\
\text { Evita jergas, abreviaturas innecesarias, } \\
\text { nombres científicos toda vez que no sea } \\
\text { necesario, usa oraciones completas, } \\
\text { evita palabras rebuscadas. }\end{array}$ \\
\hline
\end{tabular}


Tabla1 contin....

\begin{tabular}{|c|c|c|c|}
\hline \multirow{9}{*}{$\begin{array}{l}\text { Segmentos } \\
\text { Calidad del } \\
\text { cuerpo del } \\
\text { resumen }\end{array}$} & Dimensiones & Categorías & Descripción de las categorías \\
\hline & Describe el objetivo & Si/No/Dudoso & $\begin{array}{l}\text { Se menciona de manera explícita y de } \\
\text { forma clara. }\end{array}$ \\
\hline & $\begin{array}{l}\text { Objetivo y contenido } \\
\text { consistente con el título. }\end{array}$ & Si/No/Dudoso & $\begin{array}{l}\text { Objetivo del trabajo y resultado hallado } \\
\text { condice con lo expresado en el título. }\end{array}$ \\
\hline & $\begin{array}{l}\text { Objetivo consistente con el } \\
\text { título pero contenido no. }\end{array}$ & Si/No/Dudoso & $\begin{array}{l}\text { Objetivo de la investigación acorde a lo } \\
\text { expresado en título pero hallazgos } \\
\text { divergentes. }\end{array}$ \\
\hline & Tipo de resultados & Numérico & Resultados con métrica \\
\hline & & Cualitativo & Resultados en narrativo \\
\hline & & $\begin{array}{l}\text { Ambos } \\
\text { No presenta }\end{array}$ & Resultados en métrica y narrativo \\
\hline & $\begin{array}{l}\text { Presenta referencias } \\
\text { bibliográficas }\end{array}$ & $\mathrm{Si} / \mathrm{No}$ & $\begin{array}{l}\text { Se explicitan citas bibliográficas en el } \\
\text { resumen. }\end{array}$ \\
\hline & $\begin{array}{l}\text { Presenta descriptores } \\
\text { congruentes con el título }\end{array}$ & $\mathrm{Si} / \mathrm{No}$ & $\begin{array}{l}\text { Como palabras clave o en ficha } \\
\text { catalográfica. }\end{array}$ \\
\hline
\end{tabular}

Fuente: Adaptado de Paravic et al. (11), Sanabria et al. (8), Mandujano et al., (9) y Bobenrieth (10).

Como unidad de análisis se consideró el resumen de las tesis de maestrías y doctorados que han sido sustentadas para optar por el título de Máster o Magister o Doctor (PhD), por estudiantes de las Unidades académicas e Institutos de la UNA durante el periodo 20002017 que ascendió a 495. Los criterios de inclusión fueron: que las tesis hayan sido depositadas en la Biblioteca Central de la Universidad Nacional de Asunción (UNA) y que hayan sido sustentadas en el periodo de análisis, es decir, 2000 hasta 2017. Se realizó un muestreo estratificado proporcional según cantidad de tesis depositada por cada facultad e instituto (Tabla 2), siendo el tamaño muestral calculado de 164 resúmenes.

Tabla 2: Cantidad de tesis depositadas en Biblioteca Central de la UNA. Población y muestra

\begin{tabular}{lrr}
\hline Unidad Académica & $\begin{array}{r}\text { Población } \\
\text { (N) }\end{array}$ & $\begin{array}{r}\text { Muestra } \\
\text { (n) }\end{array}$ \\
\hline Facultad de Ciencias Agrarias & 117 & 48 \\
Dirección General de Postgrado-Rectorado & 99 & 42 \\
Facultad de Ciencias Exactas y Naturales & 63 & 27 \\
Facultad Politécnica & 33 & 14 \\
Facultad de Filosofía & 20 & 10 \\
Facultad de Ciencias Químicas & 14 & 14 \\
Centro Multidisciplinario de Investigaciones Tecnológicas & 8 & 5 \\
Facultad de Ingeniería & 6 & 4 \\
Total & 495 & 164 \\
\hline
\end{tabular}

Para obtener el total de tesis sustentadas, se remitió nota de solicitud a la Biblioteca Central de la UNA. Una vez disponible la información y para proceder al muestreo, se seleccionó de manera aleatoria de la lista proporcionada utilizando el código asignado por la Biblioteca a cada tesis, a fin de evitar individualizar el autor y el o los tutores del trabajo, siendo en consecuencia un enmascaramiento simple.

La evaluación de los resúmenes se realizó mediante una lista de cotejo elaborada teniendo como base los trabajos de Bobenrieth ${ }^{(10)}$, Paravic et al., ${ }^{(11)}$, Sanabria et al., ${ }^{(8)}$ y Mandujano et al.,(9) compuesta por 17 ítems, donde los cuatro primeros ítems (Tipo de programa realizado, Temas según áreas de las ciencias, Tipo de resumen y Tipo de Diseño), corresponden a caracterización del resumen y los demás (Tabla 1), evalúan la calidad. Para validar tanto el constructo como la forma, se realizó una prueba piloto con cinco resúmenes y una evaluación mediante criterios de expertos.

Para categorizar la calidad de las secciones (título y cuerpo del resumen) se consideraron cuatro niveles: Muy Bueno cuando el porcentaje promedio fue superior a 95 por ciento, Bueno entre 80 y $94 \%$, Aceptable cuando el porcentaje promedio de las dimensiones se presentaron entre 60 y 79 por ciento y No Aceptable cuando el porcentaje promedio fue inferior a 60 por ciento. 
El análisis de los datos posterior a un control de calidad se realizó en el Programa SPSS para Ciencias Sociales 17.0. Los datos se presentan en tablas de frecuencias y porcentajes y también se utilizó el estadístico Chi cuadrado $\left(X^{2}\right)$ considerando una $p<0,05$ como significativa para establecer la posible asociación entre áreas de la ciencia y tipos de resúmenes y áreas del conocimiento y método utilizado.

\section{RESULTADOS}

De 164 resúmenes de tesis analizados, $89 \%(n=146)$ correspondió a tesis para optar por el grado de maestría y $11 \%(n=11)$ para optar por el título de Doctor. La cantidad de resúmenes según año de elaboración quedó constituida de la siguiente manera: 59,1\% de los trabajos fueron elaborados entre los años 2000 y 2013 y el $40,9 \%$ de los trabajos fueron elaborados entre los años 2014-2017.

En cuanto a las áreas del conocimiento, los temas analizados tipificaban mayoritariamente $(26,1 \%)$ en el área de las Ciencias Agrícolas, seguido del área de las Ciencias Naturales $(23,7 \%)$, Ciencias Sociales $(18,2 \%)$, Ciencias Médicas y de la Salud $(15,2 \%)$, Ingeniería y Tecnología con un $12,1 \%$ de los temas analizados y en menor porcentaje $(4,2 \%)$ trabajos que caían en el área de las Humanidades.

Calidad del título

En relación a las cualidades que hacen a la calidad del título, la dimensión mejor valorada $(99,4 \%)$ fue la redacción gramatical correcta seguida del uso del tono afirmativo. En tanto la dimensión con mayor falencia $(21,9 \%)$ fue la indicación de la especificidad u objeto de investigación, donde no se visualizó o fue redactada de manera dudosa. Atendiendo que el promedio simple del porcentaje de todas las dimensiones consideradas en Calidad del Título arrojó un valor de $91,84 \%$, se calificó la redacción del título de los resúmenes como Bueno (Tabla 3).

Tabla 3: Calidad del título

\begin{tabular}{lrrrrrr}
\hline Dimensión & \multicolumn{2}{c}{ Si } & \multicolumn{2}{c}{ No } & \multicolumn{2}{c}{ Dudoso } \\
& Fr. & Por. & Fr. & Por. & Fr. & Por. \\
\hline Es indicativo del estudio & 141 & 86,0 & & & 23 & 14,0 \\
Indica la especificidad & 128 & 78,0 & 15 & 9,1 & 21 & 12,8 \\
Es conciso & 160 & 97,6 & 2 & 1,2 & & \\
Usa tono afirmativo & 161 & 98,2 & 3 & 1,8 & & \\
Es gramaticalmente correcto & 163 & 99,4 & & & 1 & 0,6 \\
\hline
\end{tabular}

Fr. (Frecuencia)/Por. (Porcentaje)

Tipo de resumen

El 78,6\% (129/164) de los resúmenes eran de tipo informativo, mientras que el $21,3 \%$ (35/164) correspondió a resumen de tipo descriptivo, es decir donde no se presentan resultados ni conclusiones. Haciendo una correlación entre las Áreas de la Ciencia, agrupadas en Ciencias Exactas y Naturales y, Ciencias Sociales y Humanidades y los tipos de resúmenes, no se encontró asociación $\left(X^{2}=0,00 ; p=0,9858\right)$ entre las áreas de las Ciencias y los tipos de resúmenes, con lo cual no es posible pensar que se den patrones al momento de redactar los resúmenes según sean los investigadores de una u otra área del conocimiento.

Tipo de investigación

El tipo de investigación predominante 54/164 (32,9\%) fue el correlacional, seguido de investigaciones descriptivas $40 / 164(24,3 \%)$ y experimental $30 / 164(18,2 \%)$ y en menor cantidad 23/164 (14,0\%) investigación cualitativa. Aquellas que de manera explícita consignaron metodología mixta (cuantitativas y cualitativas) o cuyo método o tipo de investigación no fue explicitado representaron el 10,4\% de los resúmenes. Adentrándonos en las investigaciones de tipo correlacional, del total clasificado bajo este método $(n=54)$ un $30 \%$, hace referencia a investigaciones metodológicas o teóricas donde el patrón dominante consistió en evaluar aplicabilidad de programas informáticos para resolver, mediante optimizaciones o simulaciones, el problema planteado. 
Indagando la independencia entre las áreas del conocimiento y el método utilizado, se encontró asociación entre ambas categorías $\left(X^{2}=42,70 ; \mathrm{gl}=25 ; \mathrm{p}=0,015\right)$, lo cual indica que el análisis en ciertas áreas del conocimiento conlleva ciertos métodos de análisis predominantes, como las Ciencias Sociales y el Método Cualitativo o las Ciencias Agrarias y el Experimental (Tabla 4).

Tabla 4: Cantidad de investigaciones según áreas de la Ciencia y métodos utilizados

\begin{tabular}{|c|c|c|c|c|c|c|}
\hline $\begin{array}{l}\text { Áreas de la } \\
\text { Ciencia }\end{array}$ & Descriptivo & Correlacional & $\begin{array}{l}\text { Métodos o Tipos } \\
\text { Experimental }\end{array}$ & Cualitativo & Mixto & Total \\
\hline $\begin{array}{l}\text { Ciencias } \\
\text { Naturales }\end{array}$ & 9 & 15 & 6 & 2 & 7 & 39 \\
\hline $\begin{array}{l}\text { Ingeniería y } \\
\text { Tecnología }\end{array}$ & 5 & 12 & 2 & 0 & 1 & 20 \\
\hline $\begin{array}{l}\text { Ciencias } \\
\text { Médicas y de } \\
\text { Salud }\end{array}$ & 6 & 9 & 9 & 1 & 0 & 25 \\
\hline $\begin{array}{l}\text { Ciencias } \\
\text { Agrícolas }\end{array}$ & 13 & 12 & 11 & 4 & 3 & 43 \\
\hline $\begin{array}{l}\text { Ciencias } \\
\text { Sociales }\end{array}$ & 5 & 4 & 2 & 15 & 4 & 30 \\
\hline Humanidades & 2 & 2 & 0 & 1 & 2 & 7 \\
\hline Total & 40 & 54 & 30 & 23 & 17 & 164 \\
\hline
\end{tabular}

Describe el objetivo

De los resúmenes analizados, un $82,9 \%$ mencionó el objetivo de manera explícita y un $14 \%$ no lo mencionó, en tanto un 3\% mencionó de manera muy elemental o de forma parcial. El Manual APA ${ }^{(13)}$ así como el Manual del Rectorado de la UNA ${ }^{(14)}$ manifiestan que el resumen debe contener el objetivo del trabajo, en consecuencia, atendiendo que el porcentaje de resúmenes que cumple con este requisito es mayor al $80 \%$ se calificó como Bueno.

Consistencia entre Objetivo, Resultado y Título

Se realizó una triangulación de consistencia entre aquellos resúmenes que poseían objetivo explícito, resultados informativos y el título de la investigación, donde un $68,7 \%$ si fue consistente, mientras que un $27,6 \%$ se consideró dudoso y tan solo un $3,5 \%$ de los resúmenes presentó objetivo disociado del resultado y título. Atendiendo que el porcentaje de consistencia es menor al $80 \%$, este ítem se calificó como Aceptable.

Tipo de resultados

Un 39,6 de las investigaciones presentó resultados cualitativos (narrativo), un 31,1\% resultados numéricos, el $21,3 \%$ no presentó resultados y en un $7,9 \%$ los resultados fueron numéricos y también cualitativos. Se visualizó en aproximadamente un $9 \%$ de investigaciones que aun siendo de tipo experimental presentaron resultados cualitativos. Otro aspecto identificado, fue la superficialidad con la cual se presentaron algunos resultados, lo cual no posibilita llegar a una comprensión real de los hallazgos.

Presenta descriptores congruentes con el título

Los descriptores, sea en forma de palabras clave o en ficha catalográficas congruentes con el título, se encontraron en un $79,9 \%$ de los resúmenes, mientras que un $20 \%$ de resúmenes no presentaron descriptores. Este aspecto presenta controversias en cuanto a Ios manuales; mientras el Manual APA estipula que los resúmenes deben contar con palabras clave, el Reglamento de Tesis del Rectorado de la Universidad Nacional de Asunción, no contempla explicitar los descriptores en forma de palabra clave. Considerando que el porcentaje de resúmenes que presentaban descriptores congruentes con el título prácticamente corresponde a $80 \%$, esta categoría se consideró Bueno.

Presenta referencias bibliográficas

El 95,7\% de los resúmenes no presentaba referencias bibliográficas lo cual, atendiendo a lo estipulado en el Manual APA, cuya especificación dice que los resúmenes no deben contener referencias, esta categoría se calificó como Muy Bueno, pues fue superior al 95\%. 


\section{DISCUSIÓN}

En cuanto a la redacción del título, y atendiendo que la mayor deficiencia encontrada fue la falta de la especificidad, Centty ${ }^{(18)}$ menciona que la ausencia o la explicitación difusa de la especificidad, retrasa el entendimiento a partir de la sola lectura del título, obligando al lector a realizar la prospección en segmentos posteriores como el Resumen o la Introducción del trabajo de Tesis. Esta sería una deficiencia importante en los trabajos de tesis, donde una buena construcción del título (al inicio de la investigación tentativa) "garantiza las etapas clave del proyecto, como la formulación del problema, el objetivo general y la hipótesis"(19).

Asimismo, Valarino ${ }^{(20)}$ menciona que el título de una tesis debe reflejar el tema de investigación, la especificidad es decir el qué se quiere investigar o la respuesta que se quiere dar a la pregunta de investigación, la temporalidad y la espacialidad; estos dos últimos parámetros pueden o no estar presentes dependiente de cuan determinantes sean en la investigación.

Lo hallado aquí coincide con Mandujano y Grajeda $^{(9)}$ quienes encontraron que sólo un $3,5 \%$ del título y resumen de las tesis analizadas se presentaron Excelente y un $78,5 \%$ resultó No aceptable.

En tanto los hallazgos en cuanto al tipo de investigación, donde se observó predominancia de investigaciones con niveles básicos como las descriptivas y relacionales, a decir de Umberto $\mathrm{Eco}^{(4)}$ la misma debería estar conteste con el nivel del grado pretendido, donde el aspirante a un grado de Doctor debe demostrar que puede realizar aportes de nuevos saberes a su disciplina, para lo cual resulta pertinente recurrir a tipos o métodos de trabajo que trasciendan la investigación descriptiva e incluso la correlacional, y en el caso de la investigación cualitativa, avanzar en marcos también más profundos que lo meramente descriptivo.

Una característica de las tesis es el incremento del stock de conocimiento del hombre, la cultura y la sociedad, distintos o nuevos a los ya existentes (es decir la novedad, el "novum"), logrado de manera racional y ordenada, replicable, siendo la tesis doctoral el máximo exponente de este concepto. Entre tutores, mesa examinadora y el propio tesista ha existido controversias respecto a la implicancia de "nuevo conocimiento" y sobre este punto Arias ${ }^{(21)}$ expone que nuevos conocimientos constituyen las soluciones o las respuestas a las interrogantes de los trabajos de investigación; en tanto para Mandujano y Grajeda ${ }^{(9)}$ el aporte de conocimiento se halla relacionado a la pertinencia de la investigación.

Con independencia a esta discusión, se puede coincidir que una mayor profundidad de análisis sería la que posibilita que el tesista, a decir de Viviana Mancoksky, "se vuelva creador" que construya pensamiento propio, lo fundamente y se vuelva "autor" de su obra y no reducirse a narrador de fenómenos u hechos, lo cual siguiendo a la misma autora sería la diferencia entre "ser creativo" (lo cual es otorgado por el grado de maestría) y el "ser creador" (otorgado por el doctorado) ${ }^{(22)}$.

Los resultados obtenidos donde mayoritariamente se hallaron investigaciones relacionales son superiores a lo encontrado con Tavşancıl et $a l .{ }^{(17)}$ quienes refieren que un $23,3 \%$ de los resúmenes de tesis analizados, en cuatro universidades de Turquía, fueron metodológicas y/o teóricas, y esto básicamente puede responder al hecho de haber incluido las investigaciones de tipo simulación, modelización como correlacional tipificando como investigación aplicada antes que metodológica o teórica.

En el caso de los resúmenes cuya metodología fue cualitativa, el 100\% consistió en análisis documental-descriptivo, no visualizándose otros marcos interpretativos, como el etnográfico o la investigación que haya apuntado al desarrollo de alguna teoría en un campo específico, o al análisis de alguna teoría tratando de analizar su cumplimiento o revelar inexactitudes, como lo menciona Tavşancıl(17).

Vale la pena rescatar, que mediante el análisis crítico realizado se pudo detectar que, si bien algunos resúmenes mencionaron el método de manera explícita, lo hicieron de forma superficial o insuficiente, lo cual dificultó la interpretación objetiva de la forma en que se llevó a cabo la investigación. En este sentido, Batista et al. ${ }^{(12)}$ mencionan que estudiantes y orientadores deben estar atentos al momento de redactar el método de investigación pues una descripción clara y objetiva demanda elementos esenciales para que el lector pueda comprender el desarrollo del trabajo. 
En otro orden, el haber encontrado asociación entre el método (tipo, diseño) y las áreas del conocimiento, se halla conteste en parte, con lo manifestado por Abello(23) quien dice que "la selección del método implica también la selección de un diseño; cuando es de orden cuantitativo propone acercamientos deductivos característicos de diseños experimentales, cuasi-experimentales, ex post facto y multivariados, entre otros, en tanto el método cualitativo implica acercamientos inductivos y diseños cualitativos, participativos, de casos, entre otros más propios de las Ciencias Sociales y Humanas". En consecuencia, una selección coherente del método en concordancia con el enfoque epistemológico, que va de la mano del objetivo de la investigación, permite evaluar si el investigador tiene bien fundamentado el procedimiento científico ${ }^{(23)}$.

Otro aspecto útil de rescatar, constituye el tipo de resumen. Si bien la mayoría fue de tipo informativo, es decir con mención de resultados, la calidad del dato reportado resultó exigua o muy elemental no posibilitando en algunas ocasiones, comprender los aportes. Un aspecto a considerar al momento de redactar el resumen toda vez que sea informativo, es detallar los principales hallazgos de la tesis de modo riguroso, exhaustivo, suficientemente explícito siguiendo las recomendaciones e indicaciones de las publicaciones científicas dado que el resumen define el futuro de un trabajo, es decir su relevancia ${ }^{(24,25)}$.

En tanto, el resultado hallado de consistencia entre título, objetivo y resultados es menor al reportado por Tavşancl et al., ${ }^{(17)}$ quienes mencionan que un $82,8 \%$ de los resúmenes analizados presenta consistencia entre el objetivo y el resultado y el título y un $9,9 \%$ no se presenta consistente. En tanto Mandujano y Grajeda ${ }^{(9)}$ mencionan que un estudio realizado en los Estados Unidos por Zinsmeister y Connor también encontró incongruencias entre el objetivo y las conclusiones.

Se considera que la congruencia entre objetivos y resultados debe darse en la totalidad de las tesis, empero las deficiencias podrían tener su origen en la prisa con la cual los estudiantes redactan la tesis y además, el resumen al ser la última sección redactada encuentra a los estudiantes exhaustos. Asimismo, en ocasiones, el tutor o los docentes que participan como jurado o evaluadores de la tesis no realizan aportes que puedan mejorar esta sección focalizándose en la parte textual del trabajo.

Frente a lo hallado en la investigación, se considera debe darse una mejoría en la redacción de los resúmenes, sin embargo, la evaluación de la calidad se ve dificultada dado que la Universidad Nacional de Asunción no dispone de criterios armonizados, por lo cual líneas posteriores de investigación podrían focalizar la atención en desarrollar indicadores que permitan hacerlo.

\section{REFERENCIAS BIBLIOGRÁFICAS}

1. Garay R. Estudios de Postgrados en el Paraguay. Aspectos relevantes de la dinámica de la Universidad Nacional de Asunción. Asunción: IESALC-UNESCO; 2004.

2. UNA: Universidad Nacional de Asunción. Anuario Estadístico 2017. Asunción: Universidad Nacional de Asunción; 2018.

3. CONES: Consejo Nacional de Educación Superior. Resolución 700 del 11 de noviembre 2016 Por la cual se regulan los procesos de aprobación y habilitación de los programas de postgrado. Asunción: Consejo Nacional de Educación Superior.

4. Eco U. Cómo se hace una tesis. Técnicas y procedimientos de estudio, investigación y escritura. Madrid: Gedisa; 2004.

5. De Larrucea JR. Introducción a la investigación. En: O, Amat, A, Rocafort,(Dir).Cómo investigar. Trabajo de fin de grado, tesis de máster, tesis doctoral y otros proyectos de investigación. España: PROFIT; 2017.p. 11-26.

6. UNE: Asociación Española de Normalización y Certificación. Norma Española (UNE). (Internet). Madrid: UNE. 2019

Documentación. Preparación de resúmenes. (citado el 4 de mayo 2019) Disponible en https://www.ugr.es/ anamaria/GesITS/Nor maResumenUNE_50103=1990.pdf

7. Tapia M, Rivera MC, Mora S, Barros CI. Análisis de la calidad de los resúmenes de tesis de grado de las universidades del Ecuador respecto a Normas Internacionales. Revista Ciencias de la Documentación. 2017;3 (3):40-51

8. Sanabria HA, Tarqui $C B$, Zárate $E E$. Calidad de la tesis de maestría en temas de salud pública. Estudio de una universidad pública de Lima, Perú. Educ Med.2011;14(4):215-20.

9. Mandujano Romero, E, Grajeda P.Calidad de las tesis para obtener el título de médico cirujano.Universidad Nacional de San Antonio Abad del Cusco-Perú, 20002009. Acta Med Per.2013;30(82):70-4.

10. Bobenrieth MA. Normas para revisión de artículos originales en Ciencias de la Salud. International Journal of Clinical and Healt Psychology.2002; 2(3): 509-23.

11. Paravic T, Burgos M. Evaluación de calidad de resúmenes de tesis de un programa de 
magíster en enfermería. Ciencia $y$ Enfermería.2009;15 (3): 55-68.

12. Batista MO, Paiva LM, Gómez E, Coelho M, Leite de Araujo T. Análise crítica dos resumos de teses de um Programa de Postgraduaçâ o. Acta Paul Enferm.2007; 20(2): 186-90.

13. APA: American Psychological Association. Manual de Estilo APA. (citado el 30 abril 2019.). Disponible

en http://owl.english.purdue.edu/owl/resourc e/560/01

14. UNA: Universidad Nacional de Asunción (Internet). Asunción: UNA. 2013 (citado 30 abril 2019) Por la cual se aprueba el Reglamento y Manual para la elaboración y presentación de tesis de maestrías y doctorados. Disponible en https://www.una.py/images/stories/Postg radoRRII/Manual_para_Tesis_2013.pdf

15. ABNT: Associaçao Brasileira de Normas Técnicas (ABNT).Brasil: ABTN.2019. (citado 2 de mayo 2019). Disponible enhttp://www.abnt.org.br/

16. De-Miguel M. La evaluación de tesis doctorales. Propuesta de un modelo. RELIEVE. 2010;16(1): 1-1817.

17. Tavşancıl E, Gözen G, Kezer F. Research Assistant M.A. Educational Research Association The International Journal of Educational Researchers.2011;3(1): 17-32

18. Centty DB. Manual Metodológico para el investigador científico. (internet).2006. (citado 27 abril de 2019). Disponible en www.eumed.net/libros/2010e/816/

19 Simancas ESP. Metodología para la presentación de trabajos de investigación: 2da. ed., Cartagena de la Indias: Institución Tecnológica Colegio Mayor de Bolívar; 2015.

20. Valarino E. Tesis a tiempo. Barcelona: Carnero; 2000.

21. Arias F. El proyecto de investigación. Introducción a la metodología científica. Caracas: Episteme; 2012.

22. Mancoksky V. ¿Qué se espera de una Tesis de doctorado? Breve introducción sobre algunas cuestiones y expectativas en torno a la formación doctoral. Revista Argentina de Educación Superior. 2009; 1(1): 201-16.

23. Abello R. La investigación en ciencias sociales: Sugerencias prácticas sobre el proceso. Investigación y Desarrollo. 2009; 17(1): 208-29.

24. Díez M BL. El resumen de un artículo científico: Qué es y qué no es. Investigación y Educación en Enfermería. 2007; 25(1): 14-7.

25. López CB, Alfonso IL, Armenteros I. (Comp.). Redacción y Edición de Documentos. La Habana: Editorial Ciencias Médicas; 2011. 